[Technical Paper]

\title{
Heat Dissipation Measurement of Electronic Component on PCB by Means of External Heater and Heat Flux Sensor
}

\author{
Zidi Li $^{1 *}$, Joao Vitor Thomsen Silveira ${ }^{1}$, Kazuyoshi Fushinobu ${ }^{1}$, Hiroki Nagamizo ${ }^{1,2}$, Ryuta Yasui ${ }^{1,2}$, \\ and Takuya Shinoda ${ }^{1,2}$ \\ ${ }^{1}$ Department of mechanical engineering, Tokyo Institute of Technology, 2-12-1 Ookayama, Meguro-Ku, Tokyo 152-8550, Japan \\ ${ }^{2}$ DENSO CORPORATION, 1-1-1 Shōwachō, Kariya-shi, Aichi 448-8661, Japan
}

(Received June 14, 2021; accepted October 28, 2021, published November 19, 2021)

\begin{abstract}
A new heat dissipation measurement method based on an external heater and a heat flux sensor was proposed. The information of boundary conditions and thermal properties of materials, such as the thermophysical properties of printed circuit board (PCB), in the heat transfer path is not necessary for heat dissipation measurement in the proposed method. Numerical and experimental study was carried out on testing board with single component to estimate the feasibility of this method, in addition, the effects of the thermal resistance between heater and die/chip on the relative error of the proposed measurement method are investigated. This method is proved to be able to predict the heat dissipation from a single electronic component accurately, and has high applicable for the single BGA component.
\end{abstract}

Keywords: Heat Dissipation, External Heater, Heat Flux Sensor, Relative Error, Thermal Resistance

\section{Introduction}

As power electronics system is developing towards integration, higher density and higher operation frequencies, the operation temperature of components will be higher under the same cooling condition. In the product development phase, it is of great importance to get accurate information about heat dissipation from the electronic components in order to ensure proper thermal management. Data sheet provided by manufactures may have a large relative error compared with the real heat dissipations of components.[1] When accurate heat information is not available, using the provided data sheet may lead to overengineering or unknown risks in thermal design.[2]

The most straightfor ward heat dissipation measurement method is to measure the voltage drop across a component and the current flowing through the component by electric instruments. [3] Although the heat dissipation of a component can be easily calculated by voltage times current, there is often large error in the electrical method due to the probes delays, phase shifts between sampling channels, sampling errors, nonlinearities of converter, and digital instruments sensitive to noise. [3, 4]

*: Corresponding author

E-mail: lizidi1@hotmail.com
Calorimetric measurement method [5-7] is considered to be an efficient method to measure heat dissipation from a standalone electric device such as magnetic component, capacitor, power converter and transformer, etc. The dissipated power is measured as heat by heat flux sensors when the test chamber reaches thermal equilibrium.[8] According to literature, [9] the operation power of the tested device ranges from less than $1 \mathrm{~W}$ to thousands of watts while most of the researched devices have high heat dissipation. For the devices with operation power larger than $100 \mathrm{~W}$, the measurement error is less than $2 \%$, while the measurement error will be larger when the power of device is less than $1 \mathrm{~W}$.

For some components with Temperature Sensitive Parameter in the package, junction temperature measurement method is an alternative method.[10] $T_{\mathrm{j}}$ is measurable from the forward voltage [11] and used as an input parameter in the thermal model of the component. The accuracy of this method is affected by the component thermal model environmental dependency, material property variation among different components, and measurement error of $T_{\mathrm{j}}$ as the measurement is conducted under different environments by different users. [2] Since temperature distribution is induced by heat dissipation under a certain 
boundary condition, heat dissipation can be inversely calculated from the measured temperature distribution. Razzaq et al.[12] applied inverse thermal modelling approach to compute the iron losses in an induction motor. Shoubin et al. [13] conducted the inverse heat conduction problem in the two-dimensional unsteady heat conduction system. Shuangfeng et al.[14] developed a steady-state inverse heat conduction model and predicted the heat dissipation of a GaN chip mounted on a PCB. The accuracy of the inverse thermal calculation method depended on the number of temperature measurement points and measurement noise.[14] With many heat sources, and poorly defined material properties and cooling conditions, it is very hard to get good heat dissipation estimations with the inverse thermal calculation method.

In this research, an innovative heat dissipation measurement method of single component on PCB (printed circuit board) is proposed by utilizing an external heater and a heat flux sensor, and calculating the heat dissipation from a thermal standpoint. Thermal resistance between heater and heat source in the tested component is considered to be main reason that leads to the relative error of the proposed measurement method. This method can predict the heat dissipation of a MOSFET within 16\% error and BGA within $0.1 \%$ error, respectively.

\section{Methodology of the Testing Method}

In the proposed measurement method, heater is equipped on the top surface of the component and a heat flux sensor is put under the bottom of the PCB. Figure 1 shows the schematic diagram of a testing apparatus to implement this method. A sensor is equipped under $\mathrm{PCB}$ to record the heat flow, while the block is served as a heat dissipation enhancer to transfer heat to the experimental base. Heater and component work independently, the heat dissipation of heater can be adjusted until the measured heat flow reaches the same value as the case when component works. Heat dissipation of heater which is easy to be measured, is then considered to be the same as the heat dissipation of component.

Thermal resistance network is exploited as an electrical analogy with conduction heat transfer problem of electronic component.[2, 15, 16] For a single component mounted on PCB, the thermal resistance network is shown in Fig. 2. Heater and die generate heat independently. If $Q_{0}$ is the total heat dissipation from heat sources (heater or heat sources in MOSFET/BGA), the heat flow measured by heat flux sensors are $Q_{\text {heater }}$ and $Q_{\text {component, }}$ respectively,

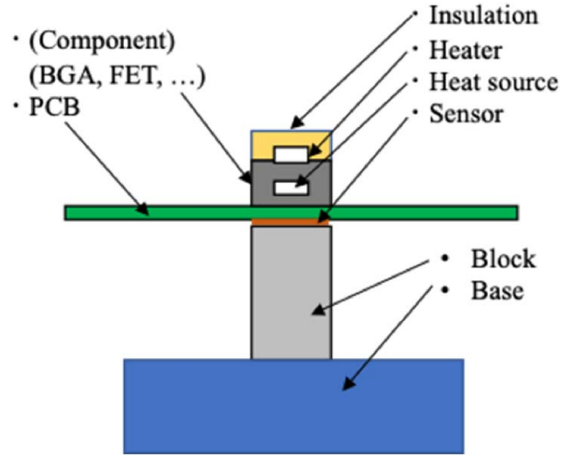

Fig. 1 Schematic diagram of the experiment setup.

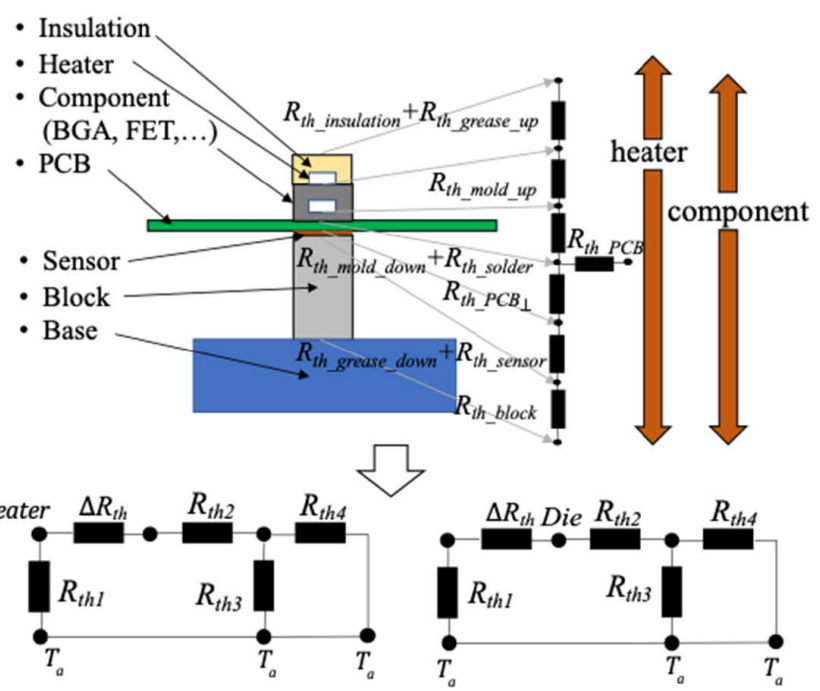

Fig. 2 Thermal resistance network of single component on a PCB.

which can be written as:

$$
\begin{aligned}
& Q_{\text {component }}=Q_{0} \times \frac{R_{t h 1} R_{t h 2}}{R_{t h 3} R_{t h 4}+\left(R_{t h 1}+R_{t h 2}\right)\left(R_{t h 3}+R_{t h 4}\right)} \\
& Q_{\text {heater }}=Q_{0} \times \frac{\left(R_{t h 1}-\Delta R_{t h}\right)\left(R_{t h 2}+\Delta R_{t h}\right)}{R_{t h 3} R_{t h 4}+\left(R_{t h 1}+R_{t h 2}\right)\left(R_{t h 3}+R_{t h 4}\right)}
\end{aligned}
$$

Where $R_{1}$ to $R_{4}$ in Eq. (1) and Eq. (2) are the sums of thermal resistances of the corresponding parts in the thermal path, and $\Delta R$ represents the thermal resistance from heater and die/chip in the component. The thermal resistances used in Eq. (1) and Eq. (2) are defined in equations as follows:

$$
\begin{aligned}
& R_{t h 1}=R_{\text {th_insulation }} \\
& R_{\text {th } 2}=R_{\text {th_mold_down }}+R_{\text {th_solder }}
\end{aligned}
$$

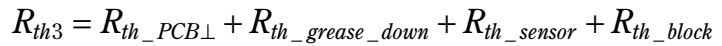

$$
\begin{aligned}
& R_{t h 4}=R_{t h \_} P C B \\
& \Delta R_{t h}=R_{t h \_ \text {mold_up }}+R_{t h_{-} \text {grease_up }}
\end{aligned}
$$

where the $R_{t h}$ represents thermal resistance of the corre- 
sponding part in the heat transfer path. $R_{t h_{-} P C B \perp}$ represents thermal resistance of $\mathrm{PCB}$ in the vertical direction,

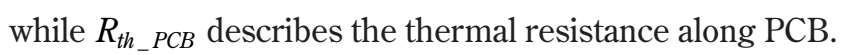
As a result, $Q_{\text {heater }}$ and $Q_{\text {component }}$ can be expressed as,

$$
\begin{aligned}
& Q_{\text {component }}=f\left(R_{t h 1}, R_{t h 2}, R_{t h 3}, R_{t h 4}, \Delta R_{t h}\right) \times Q_{0} \\
& Q_{\text {heater }}=g\left(R_{t h 1}, R_{t h 2}, R_{t h 3}, R_{t h 4}, \Delta R_{t h}\right) \times Q_{0}
\end{aligned}
$$

where $f(R)$ and $g(R)$ are only functions of thermal resistances of materials. Relative error is defined as the error between $Q_{\text {heater }}$ and $Q_{\text {component }}$, written as

$$
\begin{aligned}
& \text { Relative error }= \\
& \frac{Q_{\text {component }}-Q_{\text {heater }}}{Q_{\text {component }}}=\frac{f\left(R_{t h}\right)-g\left(R_{t h}\right)}{f\left(R_{t h}\right)}=1-\frac{1}{\eta}
\end{aligned}
$$

If the thermal resistances of materials are independent to temperature, the relative error will be a constant value. The value $\eta$ in Eq. (10) of the relative error denotes the ratio of $Q_{\text {component }}$ and $Q_{\text {heater }}$, and the Taylor series of which can be written as

$$
\eta=\frac{R_{t h 1} R_{t h 2}+R_{t h 1} \Delta R_{t h}}{R_{t h 1} R_{t h 2}+R_{t h 2} \Delta R_{t h}}=\left(1+\frac{\Delta R_{t h}}{R_{t h 2}}\right) \sum_{n=0}^{\infty}(-1)^{n} \frac{\Delta R_{t h}{ }^{n}}{R_{t h 1}{ }^{n}}
$$

It should be noted that as $R_{1}$ is the thermal resistance of insulation material, $R_{1}$ is much larger than $\Delta R$, $\lim \left(\Delta R / R_{1}\right)^{n}$ would be close to zero. Further, from Eq. (8) and Eq. (9), a certain percent of heat will flow through the sensor if the heat source is working at a certain power. It means that the percent of heat flowing through sensor can be measured by changing the power of heat sources, and relative error can be then calculated.

\section{Experimental and Numerical Study on the Com- ponents Under Test}

Experimental setup and components used in the experiment are shown in Fig. 3, a heater is put on the top surface of component, and a heat flux sensor is equipped on the bottom surface of PCB at the position of component. The testing board is mounted on a base by an aluminum block. The heater used in this research is a ceramic square heater with a size of $5 \mathrm{~mm} \times 5 \mathrm{~mm}$ and a thickness of $2 \mathrm{~mm}$. A type of BGA and MOSFET which are widely used on the ECU are considered as the testing components, the sectional views of MOSFET and BGA are shown in Fig. 4 with detailed information of the component geometrics shown in Table 1. By changing the heat dissipation of the component and heater, respectively, the corresponding heat flows transferred through heat flux sensors of the two cases are

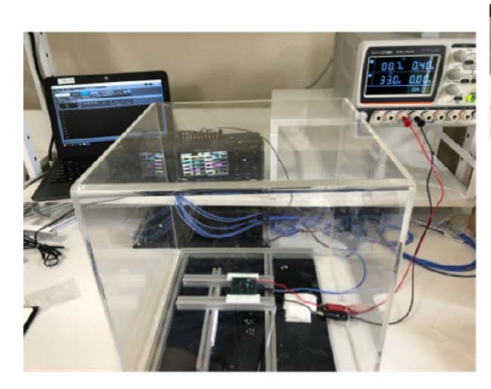

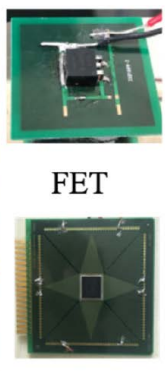

BGA

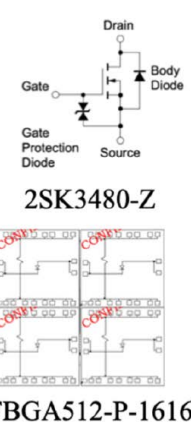

Fig. 3 Experimental setup and components used in the experiment.

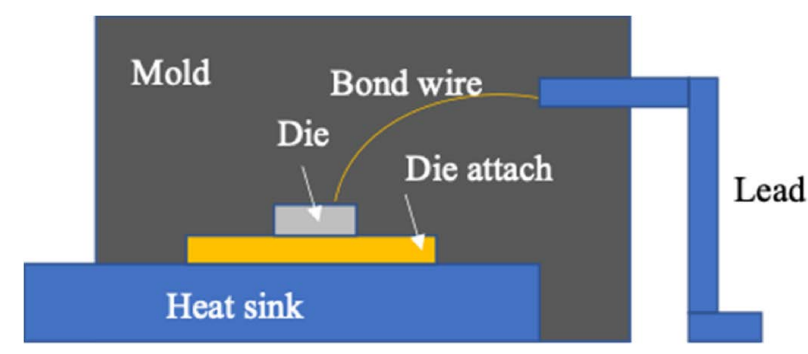

(a) MOSFET

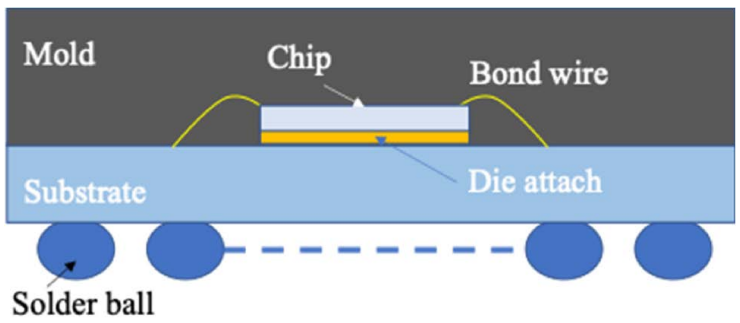

(b) BGA

Fig. 4 Schematic diagram of the section views of the packages of MOSFET and BGA.

Table 1 Geometric of components used in experiment.

\begin{tabular}{lcc}
\hline & MOSFET & BGA \\
\hline Component size [mm] & $10.0 \times 8.5$ & $16.0 \times 16.0$ \\
Mold thickness [mm] & 4.5 & 0.7 \\
PCB size [mm] & $40 \times 40$ & $100 \times 100$ \\
Die/chip size [mm] & $3.0 \times 2.2$ & $6.3 \times 6.3$ \\
\hline
\end{tabular}

recorded. Power supplies are used to drive the heater/ components, and the power dissipation of heater/components can be calculated from voltage and current. Voltage at the wire connection is used to calculate the power dissipation to avoid the voltage loss in the wire. Considering the heat dissipation range of the components under operation, the set powers of components will not exceed the normal operating powers. Each experiment is repeated for 3 times and average values are taken to reduce the experimental error.

Numerical calculations are carried out by using a com- 


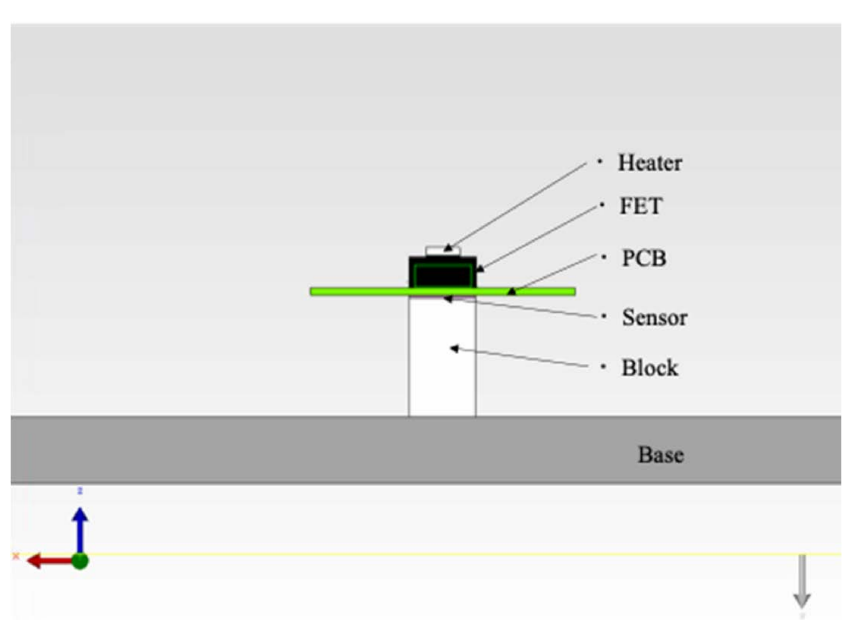

(a) MOSFET

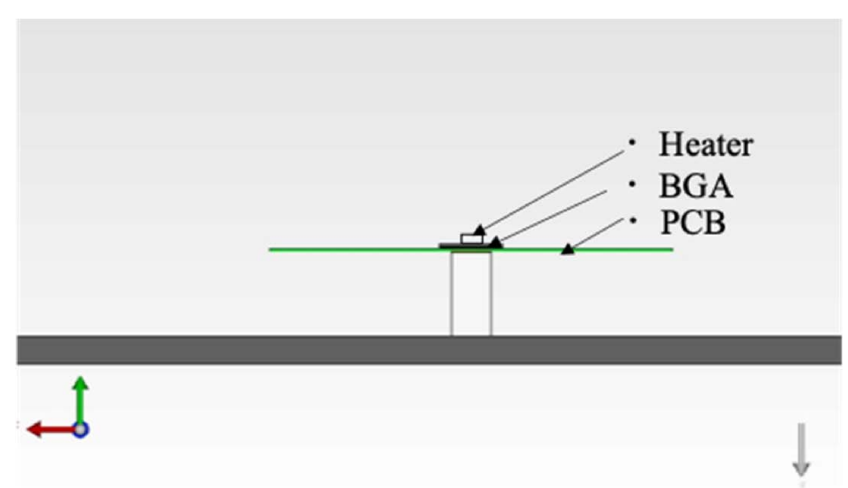

(b) BGA

Fig. 5 Numerical model of MOSFET and BGA used in simulation.

Table 2 Parameters of the materials used in the numerical model.

\begin{tabular}{lcc}
\hline & Material & $\begin{array}{c}\text { Thermal conductivity } \\
{[\mathbf{W}(\mathbf{m} \cdot \mathbf{K})]}\end{array}$ \\
\hline Mold & $\mathrm{SiO}_{2}$ & 0.81 \\
Die & $\mathrm{Silicon}$ & 117 \\
Solder & $\mathrm{SnAgCu}$ & 60 \\
Sensor & $\mathrm{SiO}_{2}$ & 0.70 \\
PCB & $\mathrm{FR} 4$ & 0.38 \\
Copper & Copper & 385 \\
Heater & Ceramic & 25 \\
\hline
\end{tabular}

mercial thermal analysis software, FloTHERM ${ }^{\circledR}$. Figure 5 shows the diagram of the simulation models, which is the same as the corresponding experimental setup shown in Fig. 3. The parameters of the materials used in the numerical model are shown in Table 2. Further, the ambient temperature is set to $25^{\circ} \mathrm{C}$ at $1 \mathrm{~atm}$, same as experiment. Grid is generated to run the simulation while much denser grid is applied to tiny parts of the model such as component and solder part. Then, the grid is adjusted so that calcu-

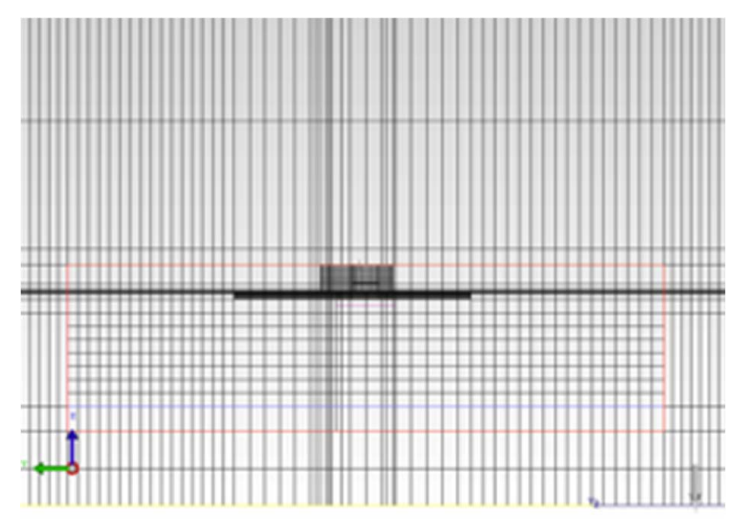

Fig. 6 Gid of FET in numerical calculation.

lated results will not change with denser grid. The grid of model of the FET is shown in Fig. 6. Total number of grids is 3,883,181 for FET model and 3,748,446 for BGA model. Same as the experiment, heat dissipations of heat sources are changed under $1.5 \mathrm{~W}$ and the heat flows through the sensors are obtained. As the position of hot spot in the heater is not clear, heat is set to be generated evenly in the heater.

\section{Experimental and Numerical Results}

The heat flow transferred through sensor is analyzed by changing the heat dissipation of each heat source experimentally and numerically. The relationship between heat flow through the sensor and the heat dissipation of heat source are shown in Fig. 7. It can be seen that the heat flows through sensors are proportional to the heat dissipations of the corresponding heat sources, which is consistent with the trend obtained from the Eq. (8) and Eq. (9) by thermal resistance network. Further, the relative errors calculated by Eq. (13) are $16.34 \%$ and $0.10 \%$ for heat dissipation measurement of MOSFET and BGA, respectively. The smaller thickness of mold between heater and chip in the BGA leads to a smaller thermal resistance $\left(\Delta R_{t h}\right)$ between heater and die/chip, which finally results in a smaller relative error in the case of BGA testing board.

Figure 8 shows the temperature distribution of the MOSFET model when heater and die works at $1 \mathrm{~W}$, respectively. The difference in the geometric sizes of die and heater leads to a different temperature field in the two cases. It can be seen that when heater works, the temperature above the heater is relatively high compared with the temperature when MOSFET works. That also implies that more heat is dissipated to ambient from component top surface and less heat is dissipated through the sensor under PCB when heater works. 


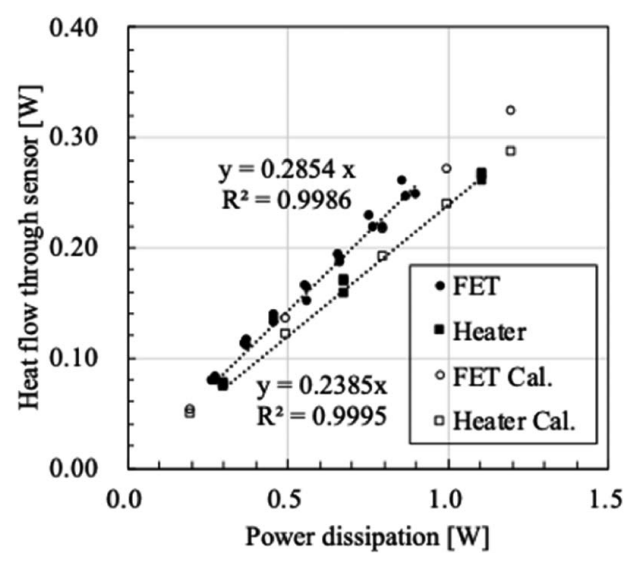

(a) MOSFET

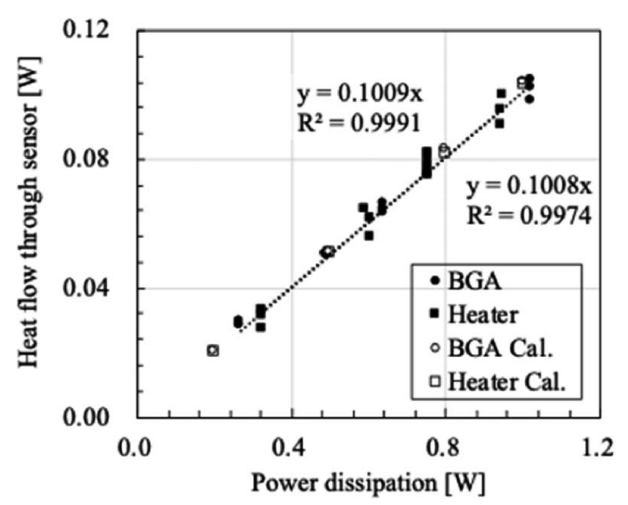

(b) BGA

Fig. 7 Experimental and numerical results of heat flow to heat dissipation.

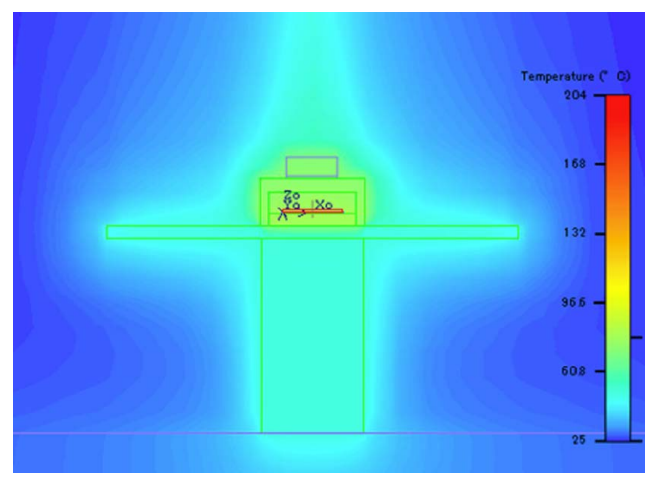

(a) MOSFET works

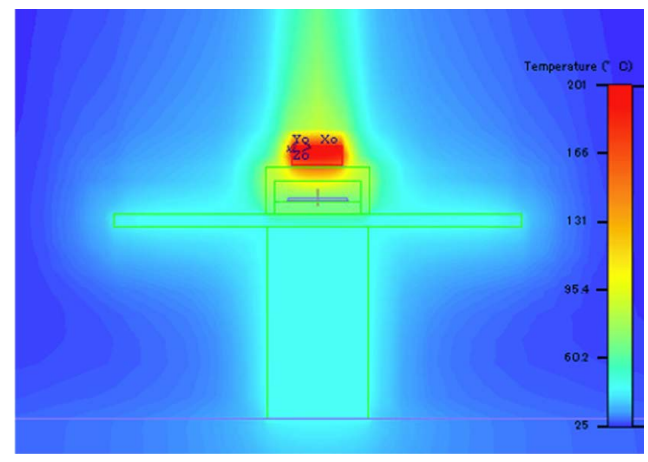

(b) Heater works

Fig. 8 Temperature distribution when each heat source works at $1 \mathrm{~W}$.

\section{Error Analysis}

In the proposed measurement method to measure the heat dissipation from components mounted on $\mathrm{PCB}$, heat flow is obtained from heat flux sensor. In reality, every measurement has error due to interaction between sensor and system, instrument error, and operation error.[17] Error analysis identifies the uncertainties in measurements and further assesses their effects on the accuracy of final results.

The heat flux sensor used in this experiment has a sensitivity of $0.0121 \mathrm{mV} /\left(\mathrm{W} / \mathrm{m}^{2}\right)$ according to the calibration report from manufacturer. Data acquisition system has a \pm $50 \times 10^{-6} \mathrm{~V}$ in accuracy. When assuming a confidence level of $95 \%$, the overall variation is $\pm 4.13 \mathrm{~W} / \mathrm{m}^{2}$ at odds of $20 / 1$, and sigma is $2.06 \mathrm{~W} / \mathrm{m}^{2}$. The film of heat flux sensor has a small thickness of $0.2 \mathrm{~mm}$, so the same amount of heat entering sensor from the PCB side will pass through the sensor from the other side. Heat flux is assumed to across the area of heat flux sensor uniformly with a nominal area of $8 \mathrm{~mm} \times 9 \mathrm{~mm}$.

Besides the error from sensor and instrumentation discussed above, the relative error which is defined by Eq. (10) and Eq. (11) should be also affected by the thermal resistance $R_{\text {th }}$ between heater and die in the component. The thermal resistance $R_{\text {th }}$ would be affected by heater size and heater positions equipped on the component because of a change in the heat transfer distance or the equivalent cross-sectional area. The schematic diagram of $R_{\text {th }}$ between heater and die is shown in Fig. 9 .

A larger heater $(10 \mathrm{~mm} \times 10 \mathrm{~mm})$ and a smaller heater $(5 \mathrm{~mm} \times 5 \mathrm{~mm})$ are used in the experiment to analyze the effects of heater size on the results of relative error of

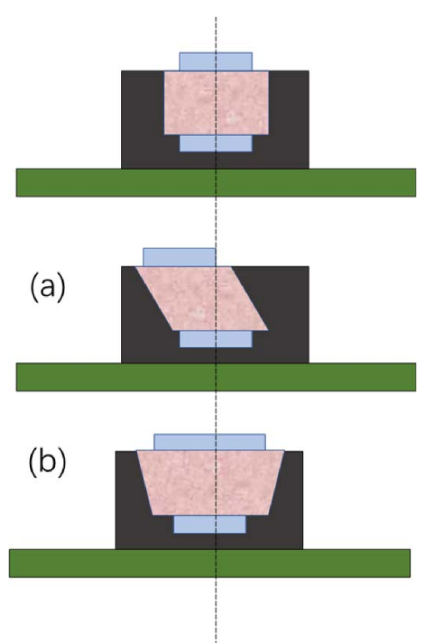

Fig. 9 Schematic diagram of $R_{\text {th }}$ between heater and die. (a) $R_{\mathrm{th}}$ with different heater position; (b) $R_{\mathrm{th}}$ with different heater size. 


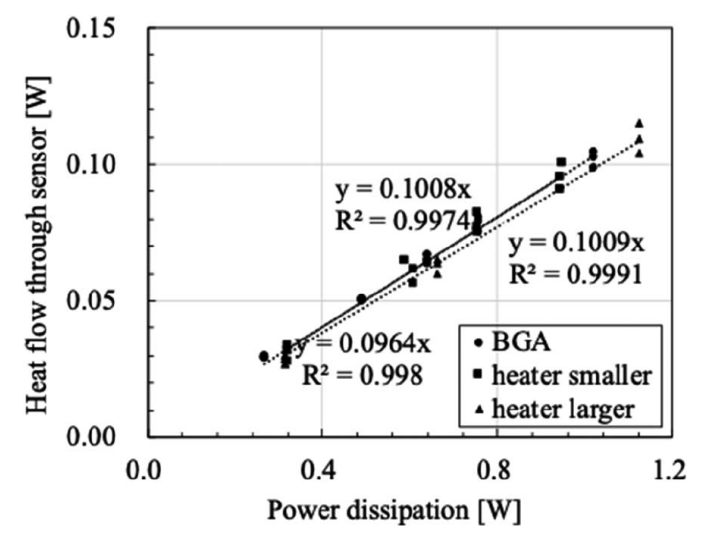

Fig. 10 Experimental results: heat flow and heat dissipation of BGA with different heater size.

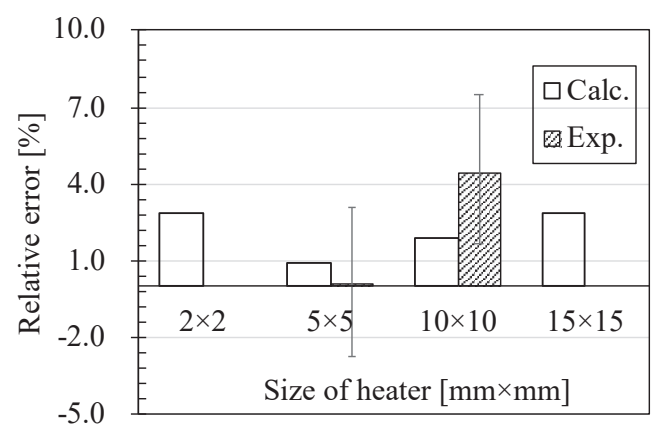

Fig. 11 relative error with different heater size.

BGA. Further, by using thermal analysis software FloTHERM, the relative error is calculated by changing heater size from $2 \mathrm{~mm} \times 2 \mathrm{~mm}$ to $15 \mathrm{~mm} \times 15 \mathrm{~mm}$. The boundary conditions in the simulation model are set to the same as the experiment, and grid is adjusted to improve the simulation accuracy. From experimental results shown in Fig. 10, less amount of heat is transferred through the sensor when using the larger heater. As a result, the relative error in the case of larger heater is $4.46 \%$, which is larger than relative error of the case with smaller heater. The calculated results show the same trend with the experimental result as shown in Fig. 11, but relative error is much larger when heater size is $2 \mathrm{~mm} \times 2 \mathrm{~mm}$, which is inconsistent with the trend. With a smaller heater size, the temperature of heater will be higher when heater is working at a same power, more heat will be dissipated to the ambient by radiation and convection, resulting in the larger relative error when a much smaller heater is used.

As heater positions will affect the thermal resistance between heater and die/chip, it is necessary to clarify the position of the heater to reduce the relative error of the proposed measurement method. In Fig. 12, different heater positions on the components are considered. The heaters are colored white while the components are black.
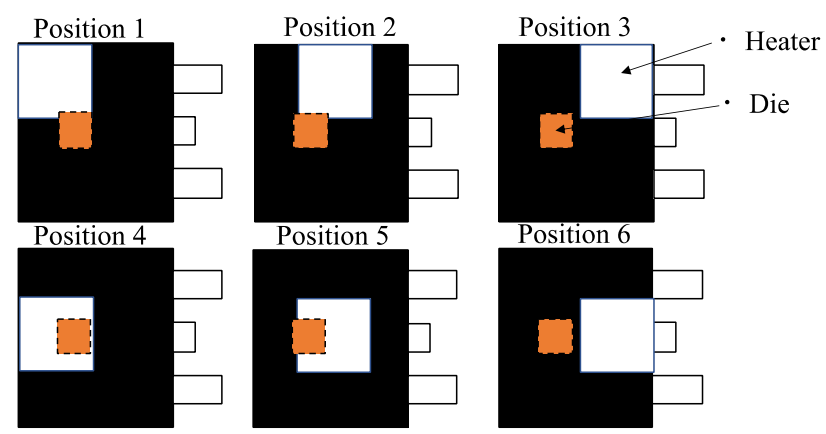

(a) MOSFET
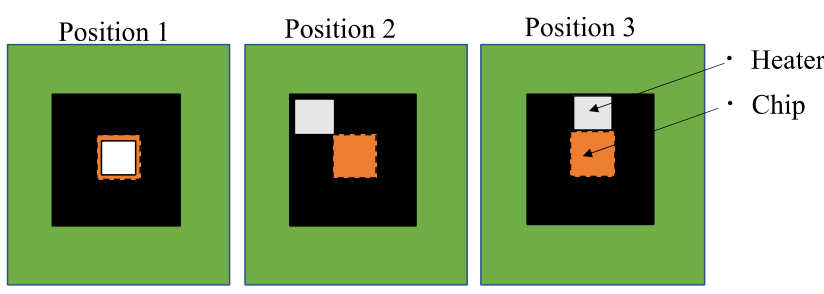

(b) BGA

Fig. 12 Different heater positions on MOSFET and BGA.

Meanwhile, locations of heat sources in components are described in Fig. 12 as die in package of MOSFET and chip in package of BGA, respectively. As the symmetry of MOSFET and BGA package, the cases in Fig. 12 (a) and Fig. 12 (b) have included all the possible heater positions on the MOSFET and BGA. Both experimental and simulation research are carried out under the cases of heater positions shown in Fig. 12. Results of the relative error of this measurement method are described in Fig. 13 when heater is placed at each position. From the results shown in Fig. 13, relative error is smallest at position 4 on MOSFET and position 1 on BGA, respectively. With a larger overlap area between heater and die/chip, the effective thermal resistance is smaller, which finally results in a smaller relative error.

As thermal resistance $R_{\mathrm{th}}$ between heater and die/chip should be the main factor that affects the relative error. The effects of $R_{\mathrm{th}}$ on the relative error of this method is examined numerically and experimentally. In the part of numerical simulation, thermal resistance $R_{\text {th }}$ between heater and die/chip which is defined by Eq. (14), is simulated by the model from Fig. 14. The simulation model follows the experimental setup used for $R_{\mathrm{th}}$ measurement in regulation JESD.[20] In the simulation model, the top surface of MOSFET is mounted on a cooling plate of a constant temperature of $25^{\circ} \mathrm{C}$. An insulator is put on the bottom surface of $\mathrm{PCB}$.

$$
R_{t h}=\frac{T_{d i e}-T_{c p}}{Q}
$$

Thermal resistance $R_{\mathrm{th}}$ can be measured experimentally 
by using structure function method.[18, 19] The heating temperature is switched off after the device reached thermal equilibrium. Cooling curve of the junction temperature $T_{\mathrm{j}}$ of Device Under Test (DUT) can be calculated from the voltage change measured by temperature sensitive parameter (TSP) during the cooling process. The structure function can be then calculated from cooling curve

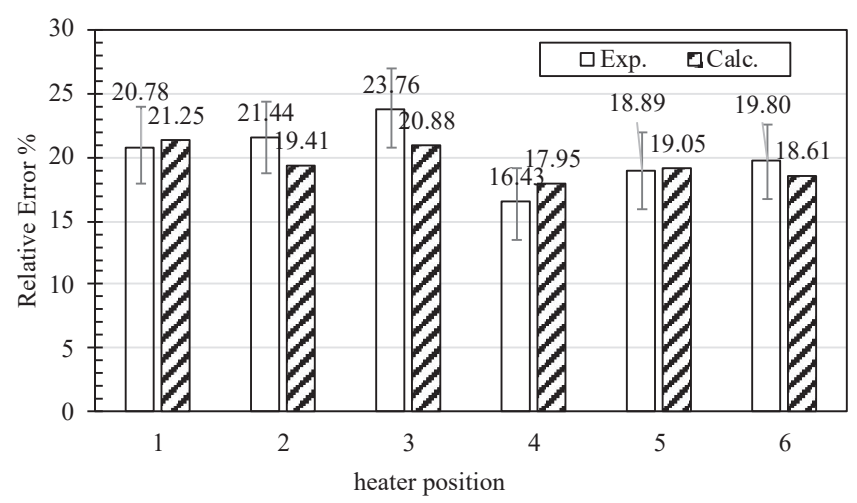

(a) FET

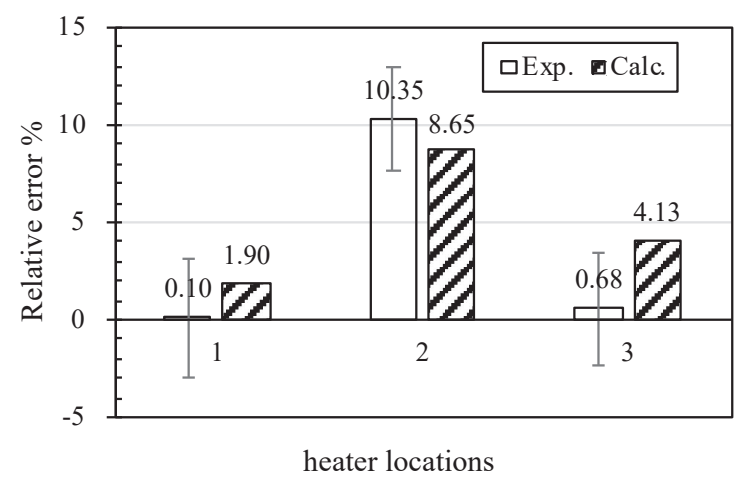

(b) BGA

Fig. 13 Relative error at different heater locations.

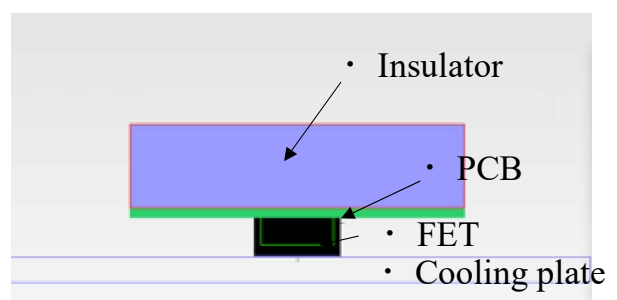

Fig. 14 Simulation model to obtain $R_{\mathrm{th}}$. through convolution operation. The structure function represents the distribution of thermal capacitance along the heat flow path, where the position on this path is expressed by the cumulative thermal resistance $\Sigma R_{\theta}$ starting from the junction. The calculation process is well described in literature. [20] A transient thermal resistance analyzer (T3Ster ${ }^{\circledR}$ ) [21] that integrates these algorithms is used in thermal resistance measurement. Experimental setup is shown in Fig. 15. The $R_{\text {th }}$ measurement requires two structure function measurements of the same semiconductor device in contact with a temperature-controlled heat-sink. The first measurement is performed without any thermal interface material (TIM) between DUT and heat sink, while a thin layer of grease is applied at the interface in the second measurement. Therefore, the structure functions of the two measurements will separate at the point where the heat flow path changes, which is the case surface of the DUT. The separate point of the structure function indicates the value of $R_{\mathrm{th}}$.

To change the thermal resistance $R_{\text {th }}$ from die to heater, different thicknesses of TIMs are covered on the components as Fig. 16. The thickness of TIM is $2.0 \mathrm{~mm}$ and 0.1 $\mathrm{mm}$ respectively with a thermal conductivity of $2.0 \mathrm{~W} /$ $(\mathrm{m} \cdot \mathrm{K})$.

Structure functions of the testing MOSFETs with different thickness of TIMs are shown in Fig. 17, the thermal resistance from heater to die $R_{\text {th }}$ is measured to be 37.8 $\mathrm{K} / \mathrm{W}$ and increases to $43.6 \mathrm{~K} / \mathrm{W}$ when the $2 \mathrm{~mm}$ TIM is used.

The above two testing MOSFETs covered with different thickness TIMs are then used to analyze the effects on the relative error of the proposed method experimentally. The experimental and calculated results of the relationship between $R_{t h}$ and relative error are shown in Fig. 18. It can be seen that when using the thicker TIM, the experimental and calculated relative error will be increased due to a larger thermal resistance between die and heater. However, there is still a difference in the results between
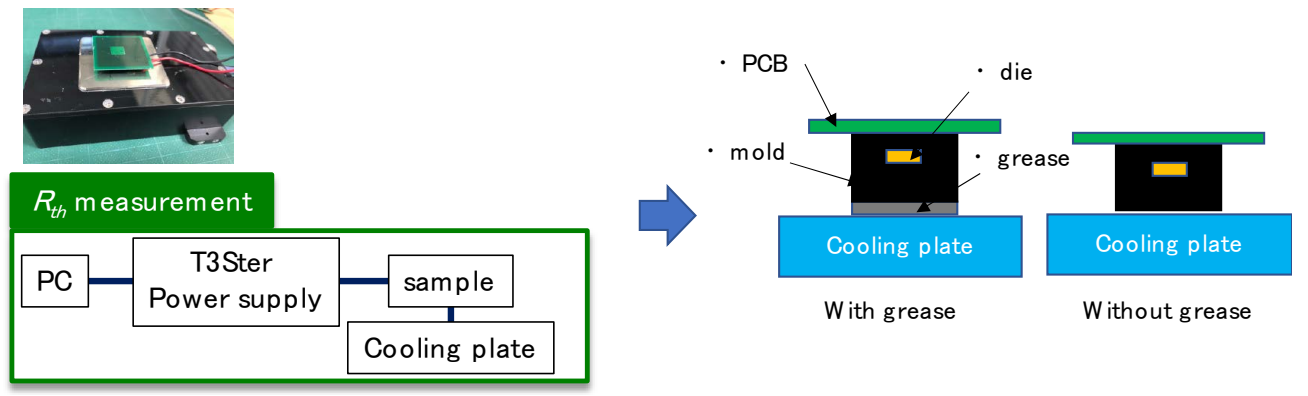

Fig. 15 Measurement of thermal resistance $R_{\mathrm{th}}$ from heater to die. 


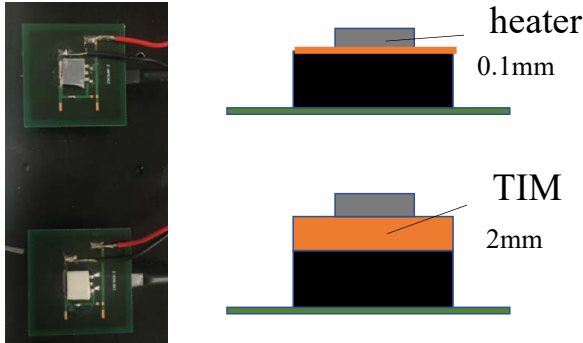

Fig. 16 Testing components with different thickness of TIM.

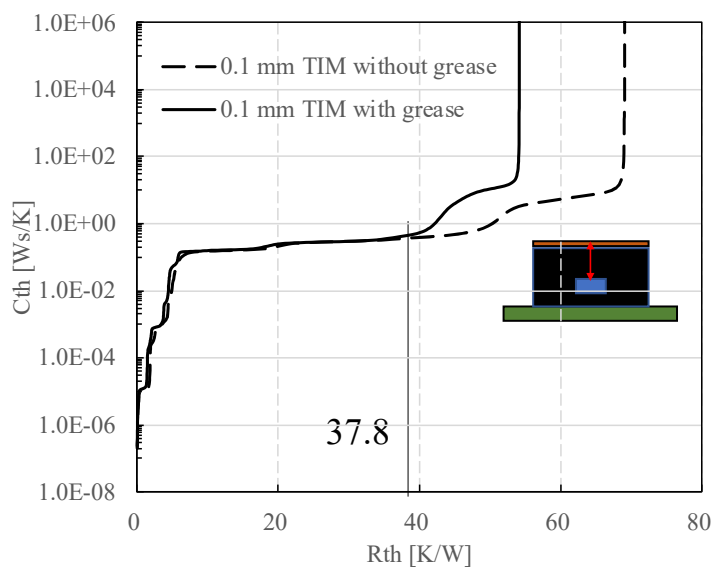

(a) MOSFET with $0.1 \mathrm{~mm}$ TIM

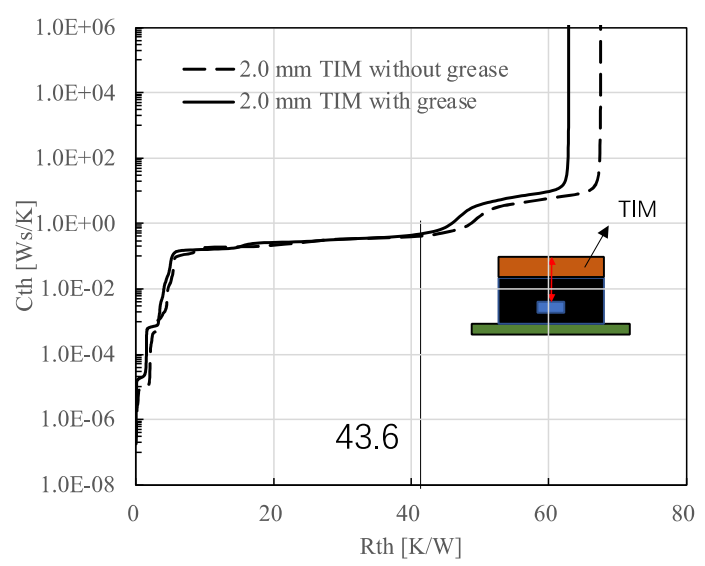

(b) MOSFET with $2.0 \mathrm{~mm}$ TIM

Fig. 17 Structure function of tested MOSFETs. (a) MOSFET with 0.1mm TIM; (b) MOSFET with $2.0 \mathrm{~mm}$ TIM.

experimental and calculated results in addition to the inherent difference between simulation model and real experimental system. For example, the contact thermal resistance is ignored in the simulation. The value of $R_{\mathrm{th}}$ at the splitting point of structure function curves is not equal to the steady-state junction to case thermal resistance defined by Eq. (14). The reason is that the steady state heat flow distribution inside the MOSFET differs from the transient heat flow distribution. Furthermore, heat is assumed to transfer through a one-dimensional heat flow path in the MOSFET in the structure function generation

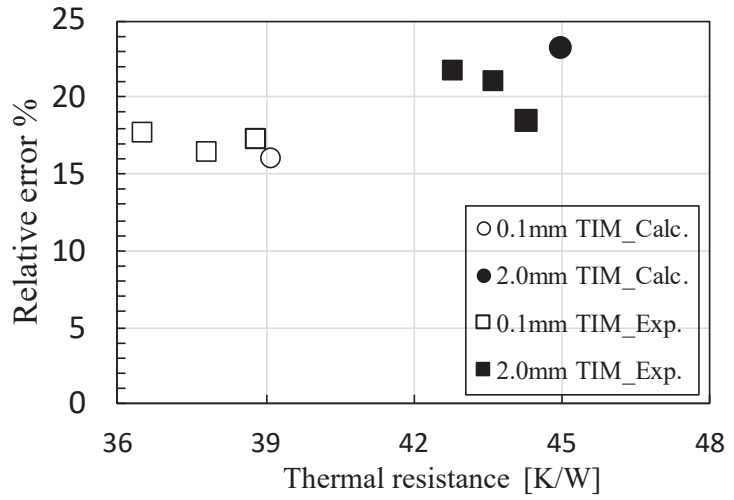

Fig. 18 Calculated and experimental $R_{t h}$ and relative error with thickness of $0.1 \mathrm{~mm}$ and $2 \mathrm{~mm}$ TIM.

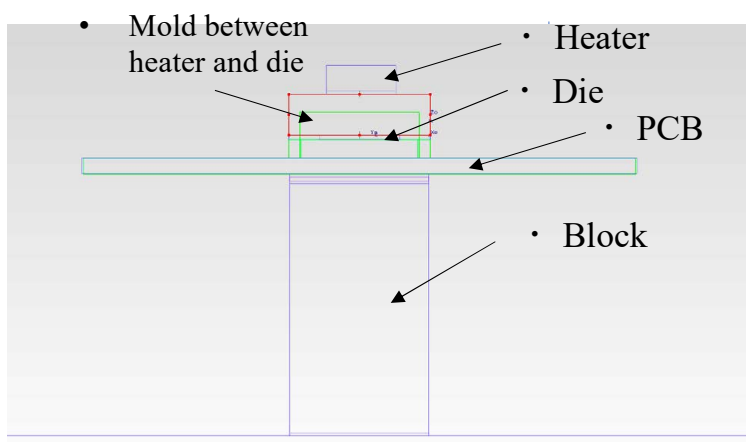

Fig. 19 Simulation model to obtain relative error.

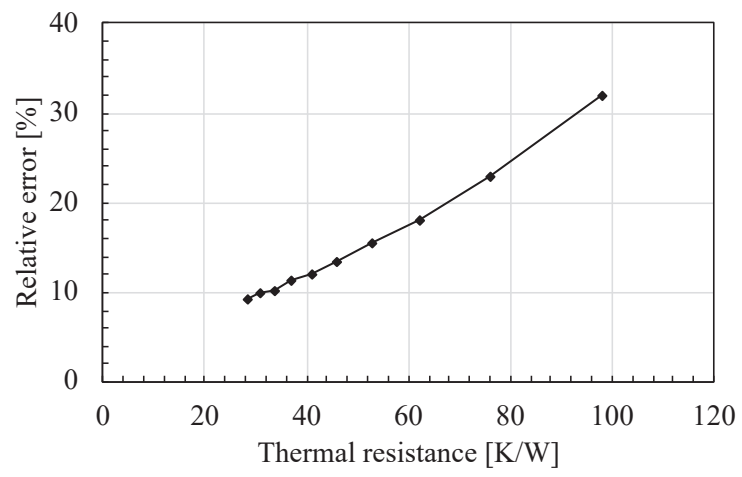

Fig. 20 Relationship between $R_{t h}$ and relative error with the change of $\lambda$ between heater and die from $0.1 \mathrm{~W} /(\mathrm{m} \cdot \mathrm{K})$ to 1.0 $\mathrm{W} /(\mathrm{m} \cdot \mathrm{K})$.

process.

To study the general trend of relative error with the change of $R_{\mathrm{th}}$, value of $R_{\mathrm{th}}$ is changed in simulation by changing the thermal conductivity, $\lambda$, of mold material between heater and die. Thermal conductivity is varied from $0.1 \mathrm{~W} /(\mathrm{m} \cdot \mathrm{K})$ to $1 \mathrm{~W} /(\mathrm{m} \cdot \mathrm{K})$ at a step of $0.1 \mathrm{~W} /(\mathrm{m} \cdot \mathrm{K})$. The simulation model is shown in Fig. 19. The calculated relationship between $R_{t h}$ and relative error is shown in Fig. 20. It is demonstrated again that relative error is reduced by reducing the thermal resistance between heater and 
die. Any methods that could reduce the thermal resistance, such as using better TIM and decreasing the thickness of mold, are conducive to reduce the relative error of the proposed measurement method.

\section{Conclusion}

We proposed a new heat dissipation measurement method of electronic components by using an external heater and a heat flux sensor. Boundary conditions and thermal properties of materials along the heat transfer path, such as the thermophysical properties of PCB, are not necessary for the method. This measurement method is carried out on a MOSFET and a BGA testing board, the respective relative errors of the testing boards are obtained. In error analysis, factors that affect the relative error is examined experimentally and numerically. Thermal resistance between heater and die/chip is considered to be the main reason that leads to the relative error. Furthermore, heater size and heater positions are clarified by measuring the relative error. The proposed method is proved to be able to predict the heat dissipation from a single electronic component accurately, and has high applicable for the single BGA component. In the future, further research would be carried out about the proposed method to measure heat dissipations from multiple electronic components on ECU.

\section{References}

[1] Okubo, Nikkei XTECH web, November 11, 2013, (in Japanese), retrieved November 16, 2020, from https://xtech.nikkei.com/dm/article/COLUMN/ 20131107/314625/

[2] Z. Qi, "A method to measure heat dissipation from component on PCB," 2012 28th Annual IEEE Semiconductor Thermal Measurement and Management Symposium (SEMI-THERM), IEEE, pp. 143-149, 2012.

[3] C. Xiao, G. Chen, and W. G. H. Odendaal, "Overview of power loss measurement techniques in power electronics systems,” IEEE Transactions on Industry Applications, Vol. 43.3, pp. 657-664, 2007.

[4] D. Iero, et al., "A calorimetry-based measurement apparatus for switching losses in high power electronic devices,” 2016 IEEE International Energy Conference (ENERGYCON), IEEE, pp. 1-5, 2016.

[5] V. Mattsson, "Comparison of calorimetric and electrical loss measurement methods in a frequency converter research and development application,” 2011
IEEE Energy Conversion Congress and Exposition, IEEE, pp. 1026-1030, 2011.

[6] P. Papamanolis, F. Krismer, and J. W. Kolar, "Minimum loss operation of high-frequency inductors," 2018 IEEE Applied Power Electronics Conference and Exposition (APEC), IEEE, pp. 1756-1763, 2018.

[7] T. Kleeb, et al., "Loss measurement of magnetic components under real application conditions,” 2013 15th European Conference on Power Electronics and Applications (EPE), IEEE, pp. 1-10, 2013.

[8] G. Chen, C. Xiao, and W. G. Odendaal, “An apparatus for loss measurement of integrated power electronics modules: Design and analysis," Conference Record of the 2002 IEEE Industry Applications Conference. 37th IAS Annual Meeting (Cat. No. $02 \mathrm{CH}$ 37344), IEEE, Vol. 1, pp. 222-226, 2002.

[9] D. Christen, et al., "Calorimetric power loss measurement for highly efficient converters,” The 2010 International Power Electronics Conference-ECCE ASIA-, IEEE, pp. 1438-1445, 2010.

[10] C. Lagarde, et al., "A new non-linear electrothermal 3D spline model with charge integration for power FETs,” 2005 European Microwave Conference, IEEE, Vol. 2, pp. 4-7, 2005.

[11] D. Schweitzer, et al., "Transient dual interface measurement-A new JEDEC standard for the measurement of the junction-to-case thermal resistance," 2011 27th Annual IEEE Semiconductor Thermal Measurement and Management Symposium, IEEE, pp. 222-229, 2011.

[12] M. Razzaq, "Loss Identification using Inverse Thermal Modelling in Cage Induction Motor,” 2019.

[13] S. Wang and R. Ni, "Solving of Two-Dimensional Unsteady-State Heat-Transfer Inverse Problem Using Finite Difference Method and Model Prediction Control Method," Complexity 2019, 2019.

[14] S. Zhang, et al., "Inverse Thermal Model of Temperature-to-Power Mapping for eGaN Systems,” PCIM Europe 2018; International Exhibition and Conference for Power Electronics, Intelligent Motion, Renewable Energy and Energy Management, VDE, 2018.

[15] Z. Chen, X. Luo, and S. Liu, "Thermal analysis of 3D packaging with a simplified thermal resistance network model and finite element simulation,” 2010 11th International Conference on Electronic Packaging Technology \& High Density Packaging, IEEE, pp. 737-741, 2010, August. 
[16] X. Luo, W. Xiong, and S. Liu, "A simplified thermal resistance network model for high power LED street lamp,” 2008 International Conference on Electronic Packaging Technology \& High Density Packaging, IEEE, pp. 1-7, 2008, July

[17] K. Azar, Thermal measurements in electronics cooling. CRC press, 1997.

[18] B. Siegal, "An alternative approach to junction-tocase thermal resistance measurements," Electronics Cooling, Vol. 7, pp. 52-57, 2001.

[19] D. Schweitzer, H. Pape, and L. Chen, "Transient measurement of the junction-to-case thermal resistance using structure functions: chances and limits,” 2008 Twenty-fourth Annual IEEE Semiconductor Thermal Measurement and Management Symposium, IEEE, pp. 191-197, 2008, March.

[20] JESD51, J. S., 14, Transient Dual Interface Test Method for the Measurement of the Thermal Resistance Junction-To-Case of Semiconductor Devices with Heat Flow through a Single Path, 2010.

[21] Measurement equipment: MicRed, “T3Ster,” Thermal transient tester, retrieved November 16, 2020, from https://www.mentor.com/products/mechanical/ micred/t3ster/

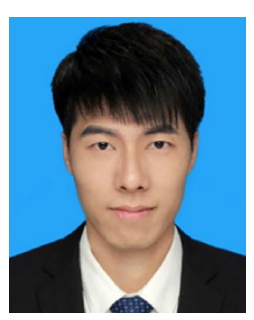

Zidi Li was born on June 2nd,1992 in Hubei province, China. He had received his Bachelor's and Master's degrees in Thermal and Energy Engineering from Beijing Institute of Technology (BIT), China, from the year 2010 to 2017. Currently, he is a PhD candidate at the Tokyo Institute of Technology (Tokyo Tech), Japan. He has worked on the field of combustion and emission control of vehicle engine. Currently, his main field of interest is in thermal management of electronic packaging.

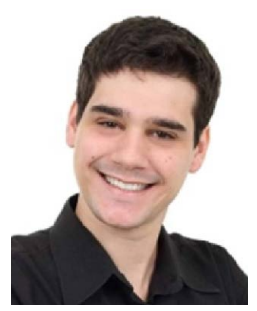

Joao Vitor Thomson Silveira was born on January 18th, 1993 in Montenegro city, Brazil. He received his Bachelor's degree (B.Sc) in Mechanical Engineering from the University of Santa Catarina in Brazil, in 2017. He got his degree of Master of Engineering in Mechanical Engineering at the Tokyo Institute of Technology, Japan, in 2020. Currently he is a $\mathrm{PhD}$ candidate at the same institute. His main field of interest is in thermal management of electronics and the surrounding disciplines required for it, such as heat transfer and fluid mechanics.

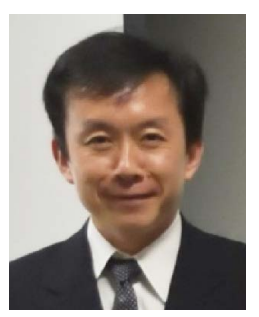

Kazuyoshi Fushinobu has received Bachelor's, Master's and Doctoral degrees in mechanical engineering from Tokyo Institute of Technology (Tokyo Tech), Japan. He is now a Research Associate and Associate Professor at Tokyo Tech. He is a Fellow of JSME. He has served as organizing members in various international academic meetings, including ISTP29 and InterPACK2019. He has worked on various research topics, including MEMS, PEMFC, energy conversion and storage technology, and is currently working on the electronic packaging, digital printing, and photonic production.

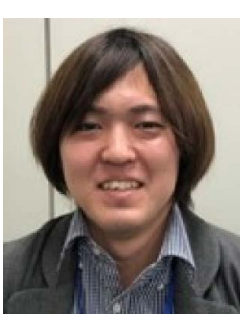

Hiroki Nakamizo was born on February 26th, 1991 in Yokohama city, Japan. He received Bachelor's degree in Electrical Engineering from Shibaura institute of technology in Japan. He was engaged in the design of automobile control computers at Persol Reserch \& Development Co., 1td, in japan, Currently, he is serving as a researcher at Tokyo Institute of Technology, in Japan. His main field of interest is in thermal management of electronics.

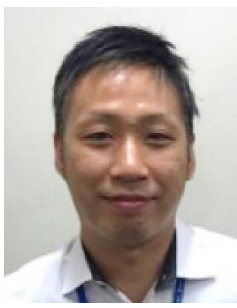

Ryuta Yasui was born on 1July18th, 1985 in Kyoto, Japan. He studied in Department of Electric Engineering of National Institute of Technology [KOSEN] Maizuru College from the year 2001 to 2006 . He currently worked in DENSO CORPORRATION in field of interests are in development and design of computers for automotive engine control and electrical thermal technologies.

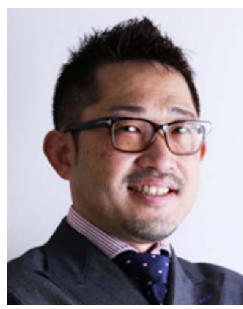

Takuya Shinoda was born on March 24th, 1967 in Aichi City, Japan. He received his Bachelor and Master degree in Department of Electric Engineering, National Institute of Technology, Oita College in Japan from the year 1982 to 1987 . Currently he is a $\mathrm{PhD}$ candidate at the Tokyo Institute of Technology (Tokyo Tech), Japan. He worked in DENSO CORPORRATION from the year 1987. His main field of interests are in development and design of computers for automotive engine control and electrical thermal technologies. He has received Best Presentation Award, Society of Automotive Engineers of Japan in the year 2019 . 